\title{
ESTUDO DE GRUPOS VIA REPRESENTAÇÃO PERMUTACIONAL
}

Luis Henrique Gabriel de Macedo, Antonio Carlos Tamarozzi

Universidade Federal de Mato Grosso do Sul, Grupo PET Matemática - Matemática, Três Lagoas , MS.

Act.ufms@gmail.com

Agência de fomento: Programa de Educação Tutorial

\section{RESUMO}

O estudo de propriedades de um Grupo pode ser feito sem a manipulação direta de seus elementos através de técnicas e ferramentas algébricas. Neste trabalho abordamos como pode ser desenvolvido o estudo de um grupo finito $G$, via representação em grupos de permutações, as chamadas representações permutacionais. A ferramenta utilizada neste processo é um homomorfismo, definido a partir de $G$ que, combinado com outras técnicas como, núcleo, grupos quociente e o Teorema de Sylow, revelam propriedades importantes de $G$, como avaliação de subgrupos e a existência de subgrupos normais. Exploramos, neste trabalho, representações permutacionais a partir de conjugações dos elementos de $G$, classes laterais e subgrupos conjugados.

Palavras-chave: grupos, subgrupos, homomorfismos, permutações, representações.

\section{STUDY GROUPS BY PERMUTATIONAL REPRESENTATIONS}

\begin{abstract}
The one group of properties study can be done without direct manipulation of its elements through technical and algebraic tools. In this paper we discuss how it can be developed the study of a finite group G, via representation on permutation groups, calls permutational representations. The tool used in this process is a homomorphism defined from $G$ which, combined with other techniques as core quotient groups and Sylow theorem reveal important properties of $\mathrm{G}$, as an evaluation subgroups and the existence of normal subgroups. We explored in this work permutational representations from combinations of elements of $G$, cosets and conjugated subgroups.
\end{abstract}

Keywords: groups, subgroups, homomorphism, permutation, representations. 


\section{INTRODUÇÃO}

Um conjunto $G$ juntamente com uma operação $*$ constitui uma estrutura algébrica $(G, *)$ que chamamos de grupo, quando se verificam as seguintes propriedades relativo a operação $*$ :

i) * é associativa em $G$;

ii) Existência do elemento neutro para * em $G$;

iii) Existência de elemento inverso para todo elemento em $G$.

Uma aplicação $\varphi$ : $G \mapsto H$ entre os grupos $(G, *)$ e $(J, \bullet)$ é chamada homomorfismo de grupos quando, para todos $x, y \in G$, tivermos $\varphi(x * y)=\varphi(x) \bullet \varphi(y)$.

A teoria dos grupos ocupa parte importante do ramo da matemática chamado álgebra abstrata, possuindo aplicações dentro e fora da matemática. Um dos maiores desafios da teoria dos grupos é o estudo de propriedades cuja execução não pode ser obtida a partir de manipulações algébricas diretas. Neste caso, técnicas alternativas são desenvolvidas para extrair resultados desejados, como por exemplo, as representações permutacionais que estudamos neste trabalho.

A partir de uma representação permutacional objetivamos inserir o grupo $G$ como subgrupo do grupo $S_{n}$, das aplicações bijetoras (permutações) do conjunto $\{1,2, \ldots, n\}$ de $n$ elementos. Esta identificação nova de $G$, combinada com outras técnicas como, núcleo, grupos quociente e o Teorema de Sylow, revelam propriedades de impacto na estrutura de $G$, em particular relacionadas à existência de subgrupos normais.

\section{METODOLOGIA}

Neste trabalho, as representações serão realizadas sobre um conjunto de permutações de um conjunto finito $C$, que será denotado por $P(C)$. Observemos que $P(C)$ consiste de todas as aplicações bijetoras de $C$ em $C$. Pode ser verificado sem dificuldades que $P(C)$ tem estrutura de grupo, mediante a operação de composição de aplicações. Na teoria dos grupos, se $\mathrm{C}$ tem $\mathrm{n}$ elementos, $\mathrm{P}(\mathrm{C})$ está identificado isomorficamente como o grupo $S_{n}$ das permutações de $n$ elementos.

Introduziremos a ferramenta principal deste trabalho, mediante a seguinte definição.

Definição: Sejam $G$ um grupo, $C$ um conjunto e $P(C)$ o grupo das permutações sobre $C$. Seja $f$ uma função de $G$ em $P(C)$. Diremos que $T$ é uma representação de $G$ em $P(C)$ se $T$ é um homomorfismo, ou seja, que $T(x y)=T(x) \circ T(y)$. Nesta situação dizemos que o grupo $G$ opera sobre o conjunto $C$.

Ao longo deste trabalho $G$ designa um conjunto com estrutura de grupo, o qual, sem perda de generalidade, assumiremos ser um grupo multiplicativo. Em consequência utilizaremos $x^{-1}$ para representar o simétrico de um elemento $x \in \mathrm{G}$ e $e_{G}$ o elemento neutro de $G$.

\section{RESULTADOS}

O exemplo mais simples de representação permutacional é um resultado conhecido como Teorema de Cayley: seja $\mathrm{G}$ um grupo finito de ordem $\mathrm{n}$; e seja $\mathrm{G}_{0}$ o conjunto $\mathrm{G}$ sem estrutura de grupo. Então a aplicação $T: G \rightarrow P\left(G_{0}\right)$, que para cada g em $\mathrm{G}$, associa a aplicação $T_{g}: G_{0} \mapsto G_{0}$ definida por $T_{g}(\mathrm{x})=g x$ é um homomorfismo injetivo. De fato Consideremos $a, b \in G$ e para qualquer $x \in \mathrm{G}_{0}$, temos $T_{a b}(x)=a b x=a(b x)=T_{a}(b x)=T_{a}\left(T_{b}(x)\right)=\left(T_{a} \circ T_{b}\right)(x)$, o que mostra ser $T e ́$ um homomorfismo. Para provar que $T$ é injetivo, consideramos:

$$
\operatorname{ker} T=\left\{g \in G / T_{g}(x)=x, \forall x \in G_{0}\right\}=\left\{g \in G / T_{g}(x)=g x=x, \forall x \in G_{0}\right\}=\left\{e_{G}\right\},
$$

e como KerT possui apenas um elemento, $T$ é injetiva .

O próximo exemplo evidencia uma representação permutacional por conjugação. Seja $G$ um grupo e seja $C=G_{0}$, onde $G_{0}$ é o conjunto $G$, sem estrutura de grupo. Consideremos:

$$
\begin{aligned}
\Psi: G & \rightarrow P(C) \\
g & \rightarrow \Psi_{g}: G_{0} \rightarrow G_{0}
\end{aligned}
$$




$$
a \rightarrow g a g^{-1}
$$

Primeiramente verificamos que $\Psi$ está bem definida, o que é trivial, pois dado $g \in G$, se $\Psi(g)=\Psi_{g}$ e $\Psi(g)=\Psi_{h}$ temos por definição que $\Psi_{g}=\Psi_{h}$

Agora basta provar que $\Psi$ é homomorfismo. Tomando $g, h \in G$ e $x \in G_{0}$ temos:

$$
\begin{gathered}
(\Psi(g h))(x)=\Psi_{g h}(x)=(g h) \times(g h)^{-1}=(g h) x\left(h^{-1} g^{-1}\right)=g\left(h x h^{-1}\right) g^{-1}= \\
=\Psi_{g}\left(h x h^{-1}\right)=\Psi_{g}\left(\Psi_{h}(x)\right)=\left(\Psi_{g} \circ \Psi_{h}\right)(x)=(\Psi(g) \circ \Psi(h))(x)
\end{gathered}
$$

Ou seja $\Psi(g h)(x)=(\Psi(g)$ o $\Psi(h))(x)$. Assim $\Psi e ́$ um homomorfismo, portanto $\Psi$ é uma representação de $G$ em $P\left(G_{0}\right)$.

\section{CORAÇÃO DE G}

A representação a seguir é um dos mais importantes exemplos do estudo aqui proposto, haja vista que permite verificar à existência de grupos normais com um numero reduzido de informações. A partir deste resultado, combinado com algumas propriedades, podemos utilizar outras ferramentas para um estudo mais profundo a respeito de conjugados e comutatividade de elementos, subgrupos e do próprio grupo.

Teorema da Representação: Seja $G$ um grupo e $H$ um subgrupo de $G$ de índice (G: $H)=n$, então $\exists N$ $\subseteq H, N \unlhd G$ tal que $G / N$ é isomorfo a um subgrupo do grupo $S_{n}$. Além disso, $N$ é o maior subgrupo normal em $G$ que está contido em $H$.

Demonstração: Sejam $S=G / H=\{H a \mid a \in G\}$ (conjunto das classes a laterais a direita de $H$ ) ,P(S) o conjunto das permutações sobre $S$, e consideremos a seguinte função:

$$
\begin{aligned}
\Psi: G & \rightarrow P(S) \\
g & \rightarrow \Psi_{g}: S \rightarrow S \\
H a & \rightarrow \mathrm{Hag}^{-1}
\end{aligned}
$$

Primeiramente precisamos verificar que está bem definida. Assim $\Psi$ independe da escolha de Ha. Se $\mathrm{Ha}, \mathrm{Hb} \in \mathrm{S}$, e $\mathrm{g} \in \mathrm{G}$ tais que:

$\Psi(g)(H a)=\Psi_{g}(H a)=H_{a g}{ }^{-1}=H b g^{-1} \Leftrightarrow a g(b g)^{-1}=a g\left(g^{-1} b^{-1}\right)=a\left(g g^{-1}\right) b^{-1}=a b^{-1} \in H \quad \operatorname{logoHa} H \mathrm{Hbe}$ assim $\Psi$ esta bem definida.

Tomando $g \in G$ e $H a, H b \in S$, se $\Psi(g)(H a)=\Psi(g)(H b)$ então:

$$
\begin{gathered}
\Psi(g)(H a)=\Psi_{g}(H a)=H a g^{-1}=H b g^{-1}=\Psi_{g}(H b)=\Psi(g)(H b) \Leftrightarrow H a g^{-1}=H b g^{-1} \Leftrightarrow \\
\Leftrightarrow H a=H b \text { logo } \Psi \text { é injetiva }
\end{gathered}
$$

Agora tomando $H a \in S$, existe $x$ tal que $\Psi(x)(H b)=H a$, basta tomar $b=a x$. Logo:

$$
\Psi(x)(H b)=\Psi(x)(H a x)=H a x x^{-1}=H a
$$

Assim $\Psi$ é sobrejetiva. Seja $g, h \in G$. Fazendo $\Psi(g h)(H a)$ temos:

$$
\Psi(g h)(H a)=H a(g h)^{-1}=H a h^{-1} g^{-1}=\Psi(g)\left(H a h^{-1}\right)=(\Psi(g) \circ \Psi(h))(H a)
$$

Assim $\Psi$ é um homomorfismo e $S \simeq S_{n}$, pois $|S|=n$. Vamos calcular o núcleo de $\Psi$ :

$$
\begin{gathered}
\operatorname{Ker}(\Psi)=\left\{g \in G / \Psi(g)=\mathrm{Hag}^{-1}=\mathrm{Ha}, \text { para todo } \mathrm{Ha} \in S\right\} \\
\Psi(g)=\mathrm{Hag}^{-1}=\mathrm{Ha} \Leftrightarrow a g a^{-1} \in H \Leftrightarrow g \in a H a^{-1}
\end{gathered}
$$

Portanto $g \in \operatorname{Ker}(\Psi) \Leftrightarrow g \in H^{a}$ para todo $a \in G$. Assim concluímos que

$$
\operatorname{Ker}(\Psi)=\bigcap_{a \in G} H^{a}
$$

Considerando $N=\operatorname{Ker}(\Psi)$, vemos que $N \unlhd G$ e $N \subseteq H$ pois $H=H^{e} \geq N$.

Agora suponhamos que exista $\mathrm{L} \unlhd \mathrm{G}$ com $\mathrm{L} \subseteq \mathrm{H}$. Como $L^{\mathrm{a}} \subseteq H^{a}$, para todo $a \in G$ logo $L \subseteq N$. Com isso concluímos que $\mathrm{N}$ é o maior subgrupo normal de $\mathrm{G}$ em $\mathrm{H}$, denominado coração de $\mathrm{H}$ em $\mathrm{G}$, e representado por $\operatorname{Core}_{\mathrm{G}}(\mathrm{H})$. Este subgrupo representa uma valiosa técnica de trabalho para a teoria dos grupos. 
Abordaremos agora o teorema de Sylow, que será utilizado como ferramenta para o estudo da ordem dos subgrupos a partir da ordem do grupo que os contém.

Utilizando o Teorema de Lagrange podemos saber quais as possíveis ordens de subgrupos de um grupo. Porém nem sempre existem subgrupos com todas as ordens indicadas pelo Teorema de Lagrange. Introduziremos um resultado que se aproxima da generalidade para este problema. Também utilizaremos sem demonstrar o corolário e os lemas abaixo, cujas demonstrações independem do teorema de Sylow.

Definição: Seja $p$ um primo e $G$ um grupo (não necessariamente finito). Dizemos que $G$ é um $p$ grupo se para todo elemento $x \in G$, a ordem de $x$ é uma potência de $p$.

Lema (Cauchy): Seja G um grupo abeliano finito. Seja $p$ um número primo que divide $|G|$. Então existe $x$ em $G$ de ordem $p$.

Corolario: Se $p^{m}$ é a maior potência de $p$ que divide $|G|$ então existe um subgrupo de $G$ com ordem $\mathrm{p}^{\mathrm{m}}$.Os subgrupos de ordem $\mathrm{p}^{\mathrm{m}}$ são chamados $\mathrm{p}$-subgrupos de Sylow.

Teorema: (Sylow): Seja $p$ um número primo, G um grupo de ordem $p^{m} b, \operatorname{com} \operatorname{MDC}(p, b)=1$, onde $m$ e $p$ são números inteiros positivos. Então para cada $0 \leq n \leq m$, existe um subgrupo $H$ em $\mathrm{G}$, tal que $|\mathrm{G}|=\mathrm{p}^{\mathrm{n}}$. Além disso, é válido que:

a) Todos os p-subgrupos de Sylow de mesma ordem são conjugados entre si. Em particular um p-subgrupo de Sylow $S$ é normal a $G$ se, e somente se, $S$ é o único subgrupo de Sylow em $\mathrm{G}$ com ordem $\mathrm{p}^{\mathrm{n}}$.

b) Se $P$ é $p$-subgrupo de $G$, então existe um $p$ subgrupo de Sylow tal que $P \subseteq S$.

c) Se $S$ é subgrupo de Sylow. Temos que o número de $p$-subgrupos de Sylow $n_{p}=$ $\left(\mathrm{G}: \mathrm{N}_{\mathrm{G}}(\mathrm{S})\right)$

d) Temos ainda que: $n_{p}$ divide $b$ e $n_{p} \equiv 1(\bmod p)$.

Lema: Seja $G$ um grupo finito e p um número primo. Sejam $S$ um p-subgrupo de Sylow de $G$ e $P$ um p-subgrupo qualquer de $G$. Então $P \cap N_{G}(S)=P \cap S$

\section{DISCUSSÃO}

Discutiremos as ferramentas exploradas neste trabalho em um exemplo particular: Aplicação: Seja G um grupo de ordem $13^{2} .11^{2}$, mostraremos que $\mathrm{G}$ é abeliano. Solução: Pelo teorema de Lagrange, temos que $|G|=|G: H| \cdot|H|$, ou seja $|H|$ divide $|G|$. Assim um subgrupo $H$ de $G$ pode ter ordens $11,11^{2}, 13$ ou $13^{2}$.

Pelo teorema de Sylow podemos concluir que existe em $G$ pelo menos um p-subgrupo de sylow $H$ de ordem $11^{2}$. Agora tentaremos descobrir o valor de $n_{11}$ sabendo que $n_{11}$ divide $13^{2}$, e $n_{11}=1(\bmod 11)$, portanto :

$$
n_{11}=1 \text { ou } n_{11}=13 \text { ou } n_{11}=13^{2}
$$

Como $13 \equiv 2(\bmod 11)$ e $169 \equiv 4(\bmod 11)$, então $n_{11}=1$ pois $1 \equiv 1(\bmod 11)$.

Logo existe apenas um subgrupo de ordem $11^{2}$ em G. Analogamente concluímos que existe pelo menos um $p$ - subgrupo de sylow $J$ de ordem $13^{2}$ e que $n_{13}$ divide $11^{2}$ e $n_{13} \equiv 1(\bmod 13)$ portanto:

$$
n_{13}=1 \text { ou } n_{13}=11 \text { ou } n_{13}=11^{2}
$$

Como $11 \equiv 11(\bmod 13)$ e $121 \equiv 4(\bmod 13)$, então $n_{11}=1$ pois $1 \equiv 1(\bmod 13)$.

Vale observar que $J$ e $H$ são subgrupos abelianos, pois todo grupo de ordem $p^{2}$ é abeliano, e que pelo teorema de Sylow são Subgrupos normais em $G$. Como $J \cap H=\left\{e_{G}\right\}$ temos que:

$J H=\{j h / j \in J, h \in H\}$ e $|J H|=13^{2} \cdot 11^{2}$, logo $G=J H$ como o produto direto de dois Grupos abelianos é abeliano, $G$ é abeliano, pois tomando $a, b \in J H$, onde $a \in J, b \in H$

$$
a b=b a \Leftrightarrow e_{g}=b a(a b)^{-1}=b a\left(b^{-1} a^{-1}\right)=\left(b a b^{-1}\right) a^{-1}=b\left(a b^{-1} a^{-1}\right)
$$

como $a \in J, J \unlhd G$ então $b a b^{-1}=a^{\prime} \in J$, e como $b^{-1} \in H, H \unlhd G$ então $a b^{-1} a^{-1}=b^{\prime}$ temos

$$
\left(b a b^{-1}\right) a^{-1}=a^{\prime} a^{-1} \in J \text { e } b\left(a b^{-1} a^{-1}\right)=b b^{\prime} \in H
$$

Assim $b a(a b)^{-1} \in J \cap H=\left\{e_{G}\right\}$, portanto $b a=a b$. 


\section{CONCLUSÃO}

A utilização de representações permutacionais em paralelo com outras técnicas da Teoria dos Grupos, como núcleo, grupos quociente e o Teorema de Sylow possibilitam a obtenção de resultados de impacto para o desenvolvimento desta teoria algébrica. Em particular demonstramos neste trabalho que, em todo grupo que admite um subgrupo $H$ de índice finito, podemos obter uma expressão para o maior subgrupo normal em $G$ que está contido em $H$, 0 chamado coração de $H$ em $G, \operatorname{Core}_{G}(H)$. Através de representações permutacionais obtém-se ainda que, o grupo quociente de $G$ pelo $\operatorname{Core}_{G}(H)$ é isomorfo a um subgrupo do grupo simétrico $\mathrm{S}_{\mathrm{n}}$.

\section{REFERÊNCIAS}

GARCIA, Arnaldo; LEQUAIN, Yves. Elementos de algebra. $5^{\text {a }}$ edição. Rio de Janeiro. IMPA, 2010.

DOMINGUES, HYGINO H.; IEZZI, G. Álgebra Moderna, São Paulo, Atual Editora LTDA, 1995.

GONÇALVES, Adilson. Introdução a Algebra. $5^{\text {a }}$ edição. Rio de Janeiro. IMPA, 2015

HERSTEIN, I. Tópicos de Álgebra, São Paulo, Ed. Polígono, 1970. 